\title{
Learning games shifting to digital
}

\author{
Andri loannou ${ }^{1}$ \\ Published online: 12 February 2021 \\ (c) Association for Educational Communications and Technology 2021
}

In the "shifting to digital" era caused by the pandemic, digital learning games seem to be a relevant topic for discussion and inquiry. While empirical literature on digital games for learning is already growing fast, the quick transition to digital is anticipated to further accelerate educators' interest in using digital games for learning. In her article "Designing and integrating purposeful learning in game play: a systematic review," Fengfeng Ke (2016) synthesized five major themes related to the design of learning games in published academic research. These were offered as guideposts for the design of future researchbased learning games.

In this special issue, we aim to present implications of scholarship on addressing current challenges related to the increased focus of digital learning. We hope that the perspectives presented herein will serve practitioners as they aim for quick transitions to online teaching and learning but also, that they will provide a starting point to further academic discussions around digital learning games design. In this special issue, we have gathered six scholarly responses to Ke's (2016) article, representing perspectives on research, evaluation, design and policy around digital learning games. The following brief synopsis of each response reveals the diversity of the responses and views on the role that gaming plays in the educational process and how digital games can be used in today's quick shifting to digital.

Victor R. Lee presents a response to the article with a focus on research, evaluation, and design. To be more responsive to the immediate needs of the educators, Lee extends the discussion of learning games published in academic research (as in Ke 2016) to include commercial learning games. As Lee explains, under limited time and pressure, many educators who appreciate games might look at commercial offerings which are more easily accessible to them compared to learning games developed at various research groups. Lee argues that Ke's work could serve the generation of a set of criteria for evaluating commercial games and presents a more comprehensible list of characteristics which can guide the evaluation of commercial games.

Alexander Nagurney gives a practical perspective on how the various themes elucidated by Ke's (2016) review of games research can support today's quick transitioning to an online format. Nagurney shifts the focus a bit to elaborate on how the lessons learned from the gaming perspective can be readily applied through a gamification lens, when a quick shift to an online modality becomes necessary. There are numerous previous discussions on how gameful

Andri Ioannou

andri.i.ioannou@cut.ac.cy

1 Dept. of Multimedia and Graphic Arts, Cyprus University of Technology, P.O. Box 50239,

3603 Limassol, Cyprus 
design and gamification have been utilized to bring game elements and fun into different types of learning activities and contexts (e.g., Ioannou 2018), yet Nagurney reframes these ideas in the context of shifting to digital.

A design perspective is presented by David Carroll Gibson who suggests that Ke's (2016) review of games research brings an opportunity to re-imagining the field of digital learning design. Gibson attempts to generalize from the five game design elements presented by $\mathrm{Ke}$ and discuss how these elements extend beyond game-based learning to other interactive learning designs such as team-based projects, collaborative problem-solving, competitions for innovative ideas, ideation-to-innovation challenges and project-based learning.

Candace Walkington brings a research perspective in the discussion and focuses her response around Ke's discussion of intrinsic integration - the notion that, in learning games, the content to be learned should be integral to the gameplay, games rules, and game actions. As Candace explains, intrinsic integration is very timely when educators seek to select games to use in their classrooms, but these games are hard to design. In her response, Candace points to a few issues that are important for the research and design of the next generation of learning games, pointing to the idea that new digital games may be increasingly designed for play in virtual settings.

Paul Barach takes a theoretical perspective and elaborates on how Ke's review of games research reveals the ongoing need for more pedagogical rigor in the design and assessment of games as well as the need for robust research on how to integrate digital games to drive meaningful learning. Barach further argues that there is a pervasive need for more experimentation and improvement of digital learning games with discussions around theories of game-based learning, integration of learning scaffolds and advancing the learner's accountability through design.

For authors, Adam Kenneth Dubé and Nicholas Joseph Dubé, Ke's (2016)'s review is seen as a comprehensive summary that can be used to guide policy on digital games in education. The authors leverage insights from Ke's (2016) review of games research in relation to the 2019 SRCD Social Policy Report on digital games, focusing on the difficulties that educators face in finding quality educational games. They present a perspective on policies aimed at helping administrators guide the adoption of games. Issues discussed include evidence-based professional development and curricula, teacher and student game consumption, and selection and purchase of effective games.

Today, the variety of available technologies to support learning and teaching is so broad that deciding what technology to use, when to use it, how to integrate it in your practice, and what outcomes to expect is a complex issue (Díaz and Ioannou 2019). This special issue aimed at guiding decisions around the integration of digital games for learning. Overall, Ke's (2016) review of games research as well as the six responses to Ke's review included in this special issue by several scholars shed light on these questions but are also calling for more inquiry on the design of games for learning. We hope that the perspectives presented herein will serve practitioners as they aim for quick transitions to online teaching and learning but also that they will provide a starting point to further academic discussions around digital learning games design, triggered by the ideas presented as future directions in the essays of our respondents.

\section{Responses in the shifting to digital special issue}

Barach, P. (2021). Designing and integrating purposeful learning in gameplay: What will it take to ensure learning games lead to sustainable learning outcomes and effectiveness? 
Dubé, A.K., \& Dubé, N. J. (2021). Policies to Guide the Adoption of Educational Games into Classrooms.

Gibson, D. (2021). Designing Purposeful Digital Learning: A comment on Ke.

Lee, V. (2021). Let's cut to commercial: Where research, evaluation, and design of learning games should go next.

Nagurney, A.J. (2021). I Have to Go Online Next week?! Practical Suggestions Based on Ke (2016).

Walkington, C. (2021). Intrinsic Integration in Learning Games and Virtual Instruction

\section{References}

Díaz, P., \& Ioannou, A. (2019). Learning in a digital world: An introduction. In P. Díaz, A. Ioannou, K. Bhagat, \& J. Spector (Eds.), Learning in a digital world. Smart computing and intelligence. Singapore: Springer.

Ioannou, A. (2018). A model of gameful design for learning using interactive tabletops: Enactment and evaluation in the socio-emotional education classroom. Educational Technology Research and Development, 67(2), 277-302.

Ke, F. (2016). Designing and integrating purposeful learning in game play: A systematic review. Educational Technology Research and Development, 64, 219-244. https://doi.org/10.1007/s1142 3-015-9418-1

Publisher's Note Springer Nature remains neutral with regard to jurisdictional claims in published maps and institutional affiliations.

Andri loannou is an Associate Professor in the Department of Multimedia and Graphic Arts of the Cyprus University of Technology, Director of the Cyprus Interaction Research Lab (https://www.cyprusinteracti onlab.com/), and Team Leader of the "Educational Media for Education and Edutainment" research group of the Research Centre on Interactive Media, Smart Systems and Emerging Technologies (CYENS). Andri has a Ph.D. in Educational Technology and an MA in Education both from the University of Connecticut (USA), and a BSc in Computer Science from the University of Cyprus. Her research contributes to key areas of educational technology, including the (i) design and evaluation of technology-enhanced learning environments, (ii) use of technology to support skills within a 21st century framework including problem-solving, collaboration, metacognition, and "living in the world" skills, (iii) design of embodied, playful and gameful learning using technology, and (iv) technology integration in all levels of education. Andri loannou has more than 80 peer-reviewed scientific publications in highly cited international journals and conferences. She has also participated in the review process of top-ranked journals in her field. She has been involved in more than 20 research projects funded by the European commission and national agencies. 